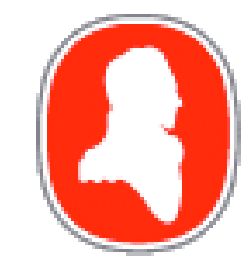

\title{
Brock University
}

Department of Computer Science

\section{A discrete duality between apartness algebras and apartness frames}

Ivo Düntsch \& Ewa Orlowska

Technical Report \# CS-08-02

January 2008

\author{
Brock University \\ Department of Computer Science \\ St. Catharines, Ontario \\ Canada L2S 3A1 \\ www.cosc.brocku.ca
}




\title{
A discrete duality between apartness algebras and apartness frames
}

\author{
Ivo Düntsch* \\ Dept of Computer Science \\ Brock University \\ St Catharines, ON, L2S 3A1, Canada \\ duentsch@brocku.ca
}

\author{
Ewa Orłowska \\ Institute of Telecommunications \\ Szachowa 1 \\ 04-894, Warszawa, Poland \\ orlowska@itl. waw.pl
}

\begin{abstract}
Apartness spaces were introduced as a constructive counterpart to proximity spaces which, in turn, aimed to model the concept of nearness of sets in a metric or topological environment. In this paper we introduce apartness algebras and apartness frames intended to be abstract counterparts to the apartness spaces of Bridges and Vîta [3], and we prove a discrete duality for them.
\end{abstract}

\section{Introduction}

A discrete duality is a duality between classes of algebras and classes of relational systems (frames): Let Alg be a class of algebras and let Frm be a class of frames. Establishing a discrete duality between these two classes requires the following steps:

1. With every algebra $A$ from Alg associate a canonical frame $\mathfrak{C} \mathfrak{f}(A)$ of the algebra and show that it belongs to Frm.

2. With every frame $X$ from Frm associate a complex algebra $\mathfrak{C m}(X)$, and show that it belongs to Alg.

3. Prove two Representation Theorems:

(a) For each $A \in \mathrm{Alg}$ there is an embedding $h: A \hookrightarrow \mathfrak{C m} \mathfrak{C} \mathfrak{f}(A)$.

(b) For each frame $X \in$ Frm there is an embedding $i: X \hookrightarrow \mathfrak{C f} \mathfrak{C m}(X)$.

Canonical frames are the counterparts of dual spaces of algebras in the Priestley style duality; however, they are not endowed with a topology and hence may be though of as having a discrete topology. Complex algebras of canonical frames are the counterparts to canonical extensions in the style of Jónsson and Tarski [8]. In the setting of discrete dualities, the canonical extension is built from the two structures which, respectively, explicitly refer to their algebraic and relational origin,. This provides an insight into the role which both algebras and frames play in establishing representation theory. A general outline of discrete duality can be found in [11].

Apartness spaces were introduced in [3] as a foundation for constructive topology in the sense of [2]. A point - set apartness is a relation $\bowtie$ between points and subsets of a set $X$ satisfying certain suitable axioms, and a

*Ivo Düntsch gratefully acknowledges support from the Natural Sciences and Engineering Research Council of Canada. He would also like to thank J. Nasr for stimulating discussions. 
pair $\langle X, \bowtie\rangle$ is called an apartness space. Intuitively, $x \bowtie S$ expresses that the point $x$ is apart from the set $S$. These relations can be considered an abstraction of metric spaces $\langle X, d\rangle$, when one sets $x \bowtie S$ if and only if $(\exists r>0)(\forall y)[y \in S \Rightarrow d(x, y)>r]$.

Apartness spaces are a natural counterpart to the proximity spaces [see 9]. Broadly speaking, a proximity on a set $X$ is a binary relation $\delta$ between subsets of $X$, and $S \delta T$ is meant to express that $S$ is near to $T$ in some sense; for a thorough treatment of proximity relations the reader is invited to consult the standard text by Naimpally and Warrack [9]. Proximity spaces have a strong connection to pointless topology and contact algebras which are of considerable interest in the field of qualitative spatial reasoning; these connections have been investigated in some detail by Vakarelov et al. [15]. A discrete duality for proximity spaces can be derived from the developments in [6].

A point - set apartness induces a point - point apartness relation $D$ on $X$ by $x D y \Longleftrightarrow x \bowtie\{y\}$, as well as an operator - defined by $-S \stackrel{\mathrm{df}}{=}\{x: x \bowtie S\}$ which behaves in some sense like complementation and which is usually called an apartness-complement. In the original papers on apartness spaces, a point-point apartness was introduced as a notion separate from the point-set apartness, but it was later shown by [14], that this is not necessary, and that $D$ will suffice. Therefore, apartness spaces can be axiomatized purely from the apartnesscomplement -.

It turns out that the apartness-complement is a sufficiency operator in the sense of Düntsch and Orłowska [5]; these were introduced in the context of modal logic to express relational properties that could not expressed with the standard possibility or necessity operators; a case in point is irreflexivity, others are antisymmetry and extensionality.

Recognizing that apartness spaces can be viewed as algebras with a sufficiency operator, one can use all the machinery developed in that area, in particular, the theory of Boolean algebras with operators. However, since we are working within an intuitionistic environment, our base structures will be Heyting algebras instead of Boolean algebras.

In this paper we introduce apartness algebras and apartness frames intended to be abstract the counterparts to apartness spaces. These two notions capture in an axiomatic way all the relevant properties of the apartness spaces and, moreover, they provide a clear division between the properties whose nature and origin are relational or algebraic. Then the discrete duality between the apartness algebras and the apartness frames clarifies how these two kinds of properties are related.

\section{Notation}

If $S \subseteq X$, we let $\bar{S}$ be the set complement of $S$ in $X$. For a function $f: X \rightarrow Y$ and $S \subseteq X$, we set $f[S] \stackrel{\text { df }}{=}\{f(x)$ : $x \in S\}$.

If $\leq$ is a partial order on $X$ and $S \subseteq X$ we define the upset $\uparrow S$ of $S$ by $\uparrow S \stackrel{\text { df }}{=}\{y:(\exists x)[x \in S$ and $x \leq y]\}$; if $S=\{x\}$, we usually just write $\uparrow x$; the collection of all upsets is denoted by $\mathscr{C}(X)$. Downsets $\downarrow S$ and $\downarrow x$ are defined analogously. Note that the complement of an upset is a downset and vice versa. The following is well known [see e.g. 13]:

Theorem 2.1. $\mathscr{C}(X)$ is a complete Heyting algebra under the set operations $\cup$ and $\cap$ and $S \rightarrow T \stackrel{\text { df }}{=} \overline{\downarrow(S \backslash T)}$.

The set of all binary relations on $X$ is denoted by $\operatorname{Rel}(X) ; 1^{\prime}$ is the identity on $X$ and $0^{\prime}$ is the diversity, i.e. $0^{\prime}=X^{2} \backslash 1^{\prime}$. If $x \in X$, we let $R(x) \stackrel{\text { df }}{=}\{z \in X: x R z\}$; the relational converse is denoted by $R^{\triangleleft}$. 
If $R \in \operatorname{Rel}(X)$, we define two modal-style operators on $2^{X}$ by

$$
\begin{aligned}
{[R](S) \stackrel{\text { df }}{=}\{x:(\forall y)[x R y \Rightarrow y \in S]\} } & =\{x: R(x) \subseteq S\} . \\
{[[R]](S) \stackrel{\text { df }}{=}\{x:(\forall y)[y \in S \Rightarrow x R y]\} } & =\{x: S \subseteq R(x)\} .
\end{aligned}
$$

[ ] is the usual necessity operator. The following correspondences are well known:

Lemma 2.2. $\quad 1 . R$ is reflexive if and only if $[R](S) \subseteq S$.

2. $R$ is transitive if and only if $[R](S) \subseteq[R][R](S)$.

[[]] is a sufficiency operator in the sense of [5], i.e.

1. $[[R]](\emptyset)=X$,

2. $[[R]](S \cup T)=[[R]](S) \cap[[R]](T)$.

Furthermore, $[[R]]$ is complete in the sense that for all $\left\{S_{i}: i \in I\right\} \subseteq 2^{X}$,

$$
[[R]]\left(\bigcup\left\{S_{i}: i \in I\right\}\right)=\bigcap\left\{[[R]]\left(S_{i}\right): i \in I\right\} .
$$

For later use, we will mention the following correspondences:

Lemma 2.3. [5]

1. $R$ is irreflexive if and only if $[[R]](S) \cap S=\emptyset$.

2. $R$ is symmetric if and only if $S \subseteq[[R]]([[R]](S))$.

$R$ is called co-transitive, if

$$
(\forall x, y)\left[x R y \Rightarrow X=R(x) \cup R^{\smile}(y)\right]
$$

The definition immediately implies

Lemma 2.4. If $R$ is not empty and co-transitive, then $\operatorname{dom}(R)=X$.

Below, we give various characterizations of co-transitivity.

Lemma 2.5. $R$ is co-transitive if and only if $[[R]](S) \subseteq[[R]](\overline{[[R]](S)})$.

Proof. This follows easily from Lemma 2.2(2) and the fact that $[R](T)=[[\bar{R}]](\bar{T})$.

Theorem 2.6. Suppose that $R$ is symmetric. Then, the following are equivalent:

1. $R$ is co-transitive.

2. $x \bar{R} y$ implies $R(x)=R(y)$.

3. $[[R]](S) \cap T=\emptyset \Rightarrow[[R]](S) \subseteq[[R]](T)$ for all $S, T \subseteq X$. 


$$
\text { 4. } x \in[[R]](S) \Rightarrow X=[[R]]\{x\} \cup[[R]](S) \text { for all } S \subseteq X \text {. }
$$

Proof. We first show 1. $\Longleftrightarrow 2$ :

" $\Rightarrow$ ": Suppose that $R$ is co-transitive, and let $x \bar{R} y$. By the symmetry of $R$ it is sufficient to show that $R(x) \subseteq R(y)$; thus, let $x R z$. Since $R$ is co-transitive and $R$ is symmetric, we have $X=R(x) \cup R(z)$. Now, $x \bar{R} y$ by the hypothesis, and thus, $y R z$, again by symmetry of $R$.

" $\Leftarrow$ ": Let $x R y$, and suppose that $z \notin R(x)$. Then, $x \bar{R} z$, and the hypothesis implies that $R(x)=R(z)$. Hence, $x R y$ implies $z R y$ and therefore, $y R z$ by symmetry.

2. $\Rightarrow 3$.: Let $[[R]](S) \cap T=\emptyset$. Choose some $x \in[[R]](X))$, i.e. $S \subseteq R(x)$; we need to show that $T \subseteq R(x)$. Let $z \in T$, and assume that $z \notin R(x)$; then, $R(x)=R(z)$ by the hypothesis. It follows that $S \subseteq R(z)$ which implies that $z \in[[R]](S)$. This contradicts $[[R]](S) \cap T=\emptyset$.

3. $\Rightarrow 4$.: Let $x \in[[R]](S)$, and $x \bar{R} y$; then, $\{x\} \cap[[R]](\{y\})=\emptyset$, and thus, $[[R]](\{y\}) \subseteq[[R]](\{x\})$ by 2. above. The symmetry of $R$ now implies that in fact $[[R]](\{y\})=[[R]](\{x\})$. Since $S \subseteq R(x)$, this shows that $S \subseteq R(y)$, and it follows that $y \in[[R]](S)$.

4. $\Rightarrow 1 .:$ Let $x R y$; then $x \in[[\{y\}]]$, and the hypothesis implies that $X=[[\{x\}]] \cup[[\{y\}]]$. Thus, $z \in R(x) \cup R(y)$ for all $z \in X$, and the symmetry of $R$ now implies co-transitivity.

We assume that the reader has some familiarity with Heyting algebras and their relatives; all unexplained lattice theoretic concepts can be found in [1].

\section{Apartness spaces}

Since we aim to work in an intuitionistic framework, our basic structures are Heyting algebras $\left\langle 2^{X}, \cup, \cap, \rightarrow, \emptyset, X\right\rangle$; the pseudocomplement of $S \subseteq X$ will denoted by $S^{*}$. If no confusion can arise, we will just use $X$ instead of the name for the full structure.

The original axiom system for apartness spaces given by Bridges and Vîta [3] involves a nonempty irreflexive and symmetric relation $D$ on a nonempty set $X$ and a relation $\bowtie$ between elements of $X$ and subsets of $X$ that satisfied certain axioms. Later, Richman [14] showed that the relation $D$ was definable from the $\bowtie$ relation, and that the original system, and that the following system was equivalent to the original one: An apartness space is a structure $\mathscr{X}=\langle X,-\rangle$, where $X$ is a nonempty set, and $-: 2^{X} \rightarrow 2^{X}$ is an operator, called an apartness complement, that satisfies $\mathrm{A}_{1}-\mathrm{A}_{5}$ below for all $x, y \in S$ and $S, T \subseteq X$ :

$\mathrm{A}_{0}$. There is some $x \in X$ such that $-\{x\} \neq \emptyset$.

$\mathrm{A}_{1} . x \in-\{y\}$ implies $y \in-\{x\}$.

A $.-S \cap S=\emptyset$.

A3. $-(S \cup T)=-S \cap-T$.

A4. $-S \cap T=\emptyset \Rightarrow-S \subseteq-T$.

A5. $x \in-S \Rightarrow X=-\{x\} \cup-S$. 
Note that $\mathrm{A}_{0}, \mathrm{~A}_{1}$ and $\mathrm{A}_{5}$ are really frame properties, since they involve singleton sets. Thus, we let $D$ be the binary relation on $X$ defined by

$$
x D y \stackrel{\text { df }}{\Longleftrightarrow} x \in-\{y\} .
$$

The $D$ - complement of $S \subseteq X$ is defined by $\sim S \stackrel{\text { df }}{=}\{x:(\forall y)[y \in S \Rightarrow x D y]\}$. By (2.2), we see that $\sim S=[[D]](S)$; thus, $\sim$ is a complete sufficiency operator, and, in particular, $\sim$ is antitone. Furthermore, - is also a sufficiency operator on $2^{X}$ by $A_{3}$ and the fact that $-\emptyset=X$ [see e.g. 3, Proposition 22].

All in all, we have three complement-like operators on $2^{X}$, and it is easy to see that

$$
-S \subseteq \sim S \subseteq S^{*}
$$

for all $S \subseteq X$. By the definition of - and $\sim$ we note that - and $\sim$ agree on singletons, i.e.

$$
x \in-\{y\} \Longleftrightarrow x \in \sim\{y\}
$$

for all $x, y \in X$.

It may be instructive to mention some topological properties derived from - and $\sim$. Since the sets of the form $-S$ are closed under finite intersections by $\mathrm{A}_{3}$, and $-\emptyset=X,-X=\emptyset$, they form the basis for a topology $\tau^{-}$on $X$ [3]. The sets of the form $\sim S$ also form the basis of a topology $\tau \sim$ in which the intersection of an arbitrary family of open sets is open; this follows from the fact that $\sim$ is a completely co-additive operator.

The decisive properties of $D$ are given by the following Lemma.

Lemma 3.1. [3] D is not empty, irreflexive, symmetric, and co-transitive.

We think of $D$ as a relation of distinctness of points. $D$ may be a proper subset of $0^{\prime}$, and thus, the fact that $x$ and $y$ are not distinct does not imply that they are equal. Note that $D$ is extensional (i.e. $D(x)=D(y)$ implies $x=y$ ) if and only if $D=0^{\prime}$.

From the characterization of co-transitivity given in Theorem 2.6(2), we observe that $\mathrm{A}_{4}$ corresponds to the separation property of proximity spaces up to extensionality of $D$ : If we think of $\bar{D}$ as a proximity of points, then $\bar{D}$ is called separated, if $x \bar{D} y$ implies $x=y$.

The sum axiom $\{x\} \delta(S \cup T) \Longleftrightarrow\{x\} \delta S$ or $\{x\} \delta T$ of proximity relations in its apartness form, however, does not follow from the axioms of apartness spaces.

The following Lemma collects some earlier results which come in helpful:

Lemma 3.2. 1. [3, Proposition 22] $-\emptyset=X$.

2. [3, Proposition 27] If $S=\bigcup_{i \in I}-S_{i}$, then $\sim S=S^{*}$. In particular, $\sim(-S)=(-S)^{*}$.

3. [3, Proposition 28] Let $x \in X$. If $(\exists T \subseteq X)[x \in-T$ and $-T \subseteq \sim S]$, then $x \in-S$.

4. [16, Lemma 2] $-S=-\sim \sim S=-\sim-S$.

In the classical case - and $\sim$ coincide:

Theorem 3.3. Suppose that ${ }^{*}$ is set complementation. Then, $\sim S=-S$. 
Proof. Clearly, $-S \subseteq \sim S$. Assume that $x \in \sim S, x \notin-S$. Now, $x \in \sim S$ if and only if $S \subseteq \sim\{x\}=-\{x\}$ by the definition of $\sim$; furthermore, $\{x\} \cap-S=\emptyset$ and $\mathrm{A}_{4}$ imply that $-S \subseteq-\{x\}$, so that altogether $S \cup-S \subseteq-\{x\}$. Now,

$$
S \cup-S \subseteq-\{x\} \stackrel{\mathrm{A}_{3}}{\Rightarrow}--\{x\} \subseteq-(S \cup-S) \stackrel{\mathrm{A}_{3}}{=}-S \cap--S \stackrel{\mathrm{A}_{2}}{=} \emptyset .
$$

On the other hand, $--\{x\} \cap-\{x\}=\emptyset$ implies $---\{x\} \cup--\{x\}=X$. The irreflexivity of $D$ implies that $x \notin-\{x\}=---\{x\}$, and thus $x \in--\{x\}$, showing that the latter is not empty.

The next result shows that $\mathrm{A}_{4}$ can be expressed as an equation using the * operator. To avoid some notational clutter, we suppose that ${ }^{*}$ binds stronger than - , i.e. we write $-S^{*}$ instead of $-\left(S^{*}\right)$.

Lemma 3.4. [7] In the presence of $\mathrm{A}_{2}$ and $\mathrm{A}_{3}$,

$$
\mathrm{A}_{4} \Longleftrightarrow-S=-(-S)^{*} .
$$

Proof. " $\Rightarrow$ ”: Since $-S \cap(-S)^{*}=\emptyset, \mathrm{A}_{4}$ implies $-S \subseteq-(-S)^{*}$.

For the converse, observe that $S \cap-S=\emptyset$ by $\mathrm{A}_{2}$ implies $S \subseteq(-S)^{*}$, and hence, $-(-S)^{*} \subseteq-S$ by $\mathrm{A}_{3}$.

" $\Leftarrow$ ": Let $-S \cap T=\emptyset$. Then, $T \subseteq(-S)^{*}$, and thus, $-(-S)^{*} \subseteq-T$, since - is antitone by $\mathrm{A}_{3}$. Our hypothesis now implies that $-S \subseteq-T$.

Next, we show that $\mathrm{A}_{5}$ follows from the other axioms:

Theorem 3.5. $\mathrm{A}_{1}-\mathrm{A}_{4}$ imply $\mathrm{A}_{5}$.

Proof. Let $x \in-S$, and suppose that $y \notin-S$; we show that $y \in-\{x\}$. Since $y \notin-S$, we have $\{y\} \cap-S=\emptyset$, i.e. $\{y\} \subseteq(-S)^{*}$. Now, observing that $-S \subseteq-(-S)^{*}$ by Lemma 3.4 - whose proof uses only $\mathrm{A}_{2}$ and $\mathrm{A}_{3}-$, we obtain

$$
\{y\} \subseteq(-S)^{*} \stackrel{\mathrm{A}_{3}}{\Rightarrow}-(-S)^{*} \subseteq-\{y\} \stackrel{\mathrm{A}_{4}}{\Rightarrow}-S \subseteq-\{y\} \Rightarrow x \in-\{y\} \stackrel{\mathrm{A}_{2}}{\Rightarrow} y \in-\{x\} .
$$

This completes the proof.

Theorem 3.6. If $R$ is a nonempty irreflexive, symmetric, and co-transitive relation on $X$, then $\langle X,[[R]]\rangle$ is an apartness space. Furthermore,

$$
[[R]](S) \cup[[R]][[R]](S)=X
$$

for all $S \subseteq X$.

Proof. Since $[[R]]$ is a sufficiency operator, $\mathrm{A}_{3}$ is satisfied, and the irreflexivity of $R$ and Lemma 2.2(1) imply $\mathrm{A}_{2}$. $\mathrm{A}_{4}$ follows from Theorem 2.6.

Finally, we show (3.3): Suppose that $x \in X$ and $S \subseteq X$, and recall that

$$
\begin{aligned}
x \in[[R]](S) & \Longleftrightarrow S \subseteq R(x), \\
x \in[[R]][[R]](S) & \Longleftrightarrow[[R]] \subseteq R(x), \\
& \Longleftrightarrow(\forall y)[S \subseteq R(y) \Rightarrow x R y] .
\end{aligned}
$$

If $x \notin[[R]](S)$, there is some $z \in S$ such that $x(-R) z$. Let $S \subseteq R(y)$; then, $z R y$, and co-transitivity of $R$ implies that $R(x)=R(z)$. It follows that $x R y$. 
Condition (3.3) enables us to express the fact that the apartness complement - is the $D$ - complement $\sim$ by an equation in -:

Lemma 3.7. If $-S \cup--S=X$ for all $S \subseteq X$, then $-=\sim$.

Proof. By (3.2), all we have to show is $\sim S \subseteq-S$; thus, let $x \in \sim S$. Then, $\{x\} \subseteq \sim S$, and it follows that $\sim \sim S \sim\{x\}=-\{x\}$ since $\sim$ is antitone.

If $x \in-S$, we are done. Otherwise, $x \in--S$; then, by $\mathrm{A}_{5},-\{x\} \cup--S=X$. Since $-S \cap--S=\emptyset$ by $\mathrm{A}_{2}$, it follows that $-S \subseteq-\{x\}$. Thus, $\sim \sim S \cup-S \subseteq-\{x\}$, and hence, $--\{x\} \subseteq-\sim \sim S \cap--S$, since - is antitone. By Lemma 3.2(4), $-\sim \sim S=-S$, and thus, $--\{x\} \subseteq-S \cap--S=\emptyset$, i.e. $-\{x\}=X$. This, however, contradicts $\{x\} \cap-\{x\}=\emptyset$.

\section{Apartness algebras}

The aim in this and the next Section is to prove a duality theorem for a class of apartness spaces, namely, those in which the apartness complement is the $D$ - complement. By Lemma 3.7, these are exactly the apartness spaces for which $-S \cup--S=X$ for all $S \subseteq X$.

With some abuse of language we will identify the structure with its universe, if no confusion is likely to arise. If $\langle L,+, \cdot \rightarrow, 0,1\rangle$ is a Heyting algebra (HA) and $a \in L$, we denote the pseudocomplement $a \rightarrow 0$ of $a$ by $a^{*}$. The collection of all prime filters of $L$ is denoted by $\operatorname{Prim}(L)$.

$a \in L$ is called dense, if $a^{*}=0$, and the set of dense elements is denoted by $\mathscr{D}(L)$. An element $a$ of $L$ is called regular, if is is of the form $b^{*}$ for some $b \in L$. The collection of regular elements of $L$ is denoted by $\mathscr{R}(L)$. An element $a$ of $L$ is called complemented, if $a+a^{*}=1$. Clearly, each complemented element is regular, and the set $C(L)$ of complemented elements of $L$ is a subalgebra of $L$ and a Boolean algebra. If $S \subseteq L$, we set $S_{C} \stackrel{\text { df }}{=} S \cap C(L)$.

The following are well known, see e.g. [1]:

Lemma 4.1. 1. $\mathscr{D}(L)$ is a filter of $L$.

2. $\left\langle\mathscr{R}(L), \vee, \wedge,{ }^{-}, 0,1\right\rangle$ is a Boolean algebra with the operations $a \wedge b=a \cdot b, a \vee b=(a+b)^{* *}$, and $\bar{a}=a^{*}$. Furthermore, $\mathscr{R}(L) \cong L / \mathscr{D}(L)$ via the assignment $a \mapsto a^{* *}$.

3. A prime filter $F$ of $L$ is maximal if and only if $\mathscr{D}(L) \subseteq F$ if and only if $(\forall a)\left[a^{* *} \in F \Rightarrow a \in F\right]$.

An apartness algebra is a Heyting algebra $\langle L,+, \cdot, \rightarrow, 0,1, \rho\rangle$ with a unary operator $\rho$ that satisfies the following conditions:

$\mathrm{HA}_{1} . \rho(0)=1$.

$\mathrm{HA}_{2} \cdot \rho(x+y)=\rho(x) \cdot \rho(y)$.

$\mathrm{HA}_{3} . \rho(x) \leq x^{*}$.

$\mathrm{HA}_{4} . x \leq \rho \rho(x)$.

$\mathrm{HA}_{5} . \rho(x)+\rho(\rho(x))=1$. 
$\rho$ is called trivial, if $\rho(x)=0$ for all $x \neq 0$.

$\mathrm{HA}_{5}$ tells us that the image of $\rho$ consists of complemented elements $\rho(x)$, in particular, that $\rho(\rho(x))=\rho(x)^{*}$, since $\rho(x) \cdot \rho(\rho(x))=0$ by $\mathrm{HA}_{3}$. Furthermore, $\mathrm{HA}_{5}$ reflects the property $[[R]](S) \cup[[R]][[R]](S)=X$ of Theorem 3.6.

If $L$ is a Stone algebra, then $\rho$ obviously satisfies $\mathrm{HA}_{5}$. On the other hand, not every apartness algebra is a Stone algebra, as the example in Figure 1 shows. There, $\rho\left(x^{*}\right)=0$, and $\rho(y)=\rho(x)$.

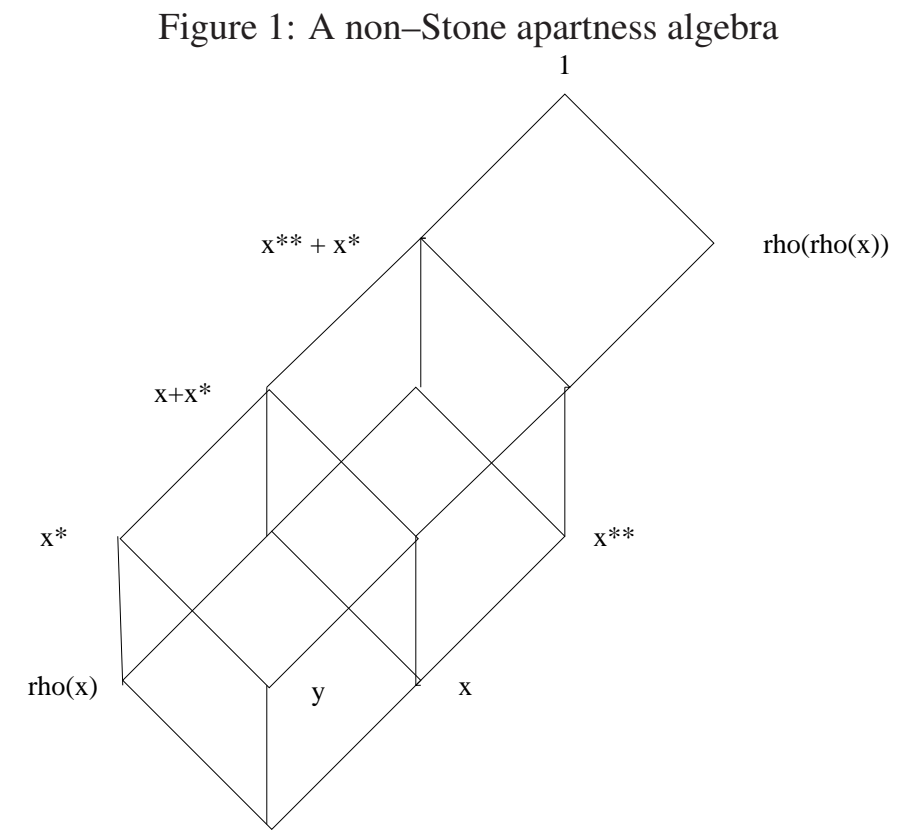

0

Let us first exhibit some simple properties of apartness algebras. By $\mathrm{HA}_{1}$ and $\mathrm{HA}_{2}, \rho$ is a sufficiency operator. Furthermore,

Lemma 4.2. 1. $\rho(x)=\rho(\rho(\rho(x)))$.

2. $x \leq y \leq \rho(\rho(x)) \Rightarrow \rho(y)=\rho(x)$.

3. If $F, G \subseteq L, G=\uparrow G$, and $F \cap \rho[G] \neq \emptyset$, then $F_{C} \cap \rho\left[G_{C}\right] \neq \emptyset$.

Proof. 1. By HA $4, \rho(x) \leq \rho(\rho(\rho(x)))$. Conversely, since $x \leq \rho(\rho(x))$ by $\mathrm{HA}_{4}$ and since $\rho$ is antitone, we have $\rho(\rho(\rho(x))) \leq \rho(x)$ by $\mathrm{HA}_{3}$.

2. This follows immediately from $\mathrm{HA}_{2}$ and 1 . above.

3. Suppose that $F \cap \rho[G] \neq \emptyset$, say, $x \in G$ and $\rho(x) \in F$. By HA $5, \rho(x), \rho(\rho(x)) \in C(L)$. Since $G=\uparrow G$ and $x \leq \rho(\rho(x))$ by $\mathrm{HA}_{4}, \rho(\rho(x)) \in G_{C}$, and $\rho(\rho(\rho(x)))=\rho(x) \in F$.

The following property corresponds to Axiom $\mathrm{A}_{4}$ of apartness spaces:

$$
\mathrm{A}_{4}^{\mathrm{alg}} \cdot \rho(x) \cdot y=0 \Rightarrow \rho(x) \leq \rho(y) .
$$

The property $\mathrm{A}_{4}^{\text {alg }}$ can be expressed equationally in various ways: 
Theorem 4.3. The following are equivalent:
1. $\mathrm{A}_{4}^{\mathrm{alg}}$.
2. $\rho(x)=\rho\left(\rho(x)^{*}\right)$.
3. $\rho(x)^{*}=\rho(\rho(x))$.

Proof. 1. $\Rightarrow 2$ :: Since $\rho(x) \cdot \rho(x)^{*}=0, \mathrm{~A}_{4}^{\text {alg }}$ implies that $\rho(x) \leq \rho\left(\left(\rho(x)^{*}\right)\right.$. Conversely, $x \cdot \rho(x)=0$ implies $x \leq \rho(x)^{*}$, and thus, $\rho\left(\rho(x)^{*}\right) \leq \rho(x)$, since $\rho$ is antitone by $\mathrm{HA}_{2}$.

2. $\Rightarrow$ 3.: Consider

$$
\mathrm{HA}_{4} \Rightarrow \rho\left(x^{*}\right) \leq \rho\left(\rho\left(\rho\left(x^{*}\right)\right)\right) \stackrel{2 .}{=} \rho(\rho(x)) .
$$

Since $\rho(x) \cdot \rho(\rho(x))=0$ by $\mathrm{HA}_{3}$, we obtain $\rho(\rho(x)) \leq \rho(x)^{*}$.

3. $\Rightarrow 1 .:$ Suppose that $\rho(x) \cdot y=0$. Since $\rho(\rho(x))=\rho(x)^{*}$, it follows that $y \leq \rho(\rho(x))$, and thus, $\rho(\rho(\rho(x))) \stackrel{4.2}{=}$ $\rho(x) \leq \rho(y)$.

Observe that $\mathrm{A}_{4}^{\mathrm{alg}}$ tells us that $\rho[L] \subseteq \mathscr{R}(L)$, and $\mathrm{HA}_{5}$ says that $\rho[L] \subseteq C(L)$. Thus, we see that $\mathrm{HA}_{5}$ implies $\mathrm{A}_{4}^{\mathrm{alg}}$. The converse, however, is not true: Consider the Heyting algebra shown in Figure 2. If $\rho(x)=x^{*}$, then $L$ satisfies

Figure 2: An algebra that satisfies $\mathrm{HA}_{1}-\mathrm{HA}_{4}$ and $\mathrm{A}_{4}^{\text {alg }}$, but not $\mathrm{HA}_{5}$

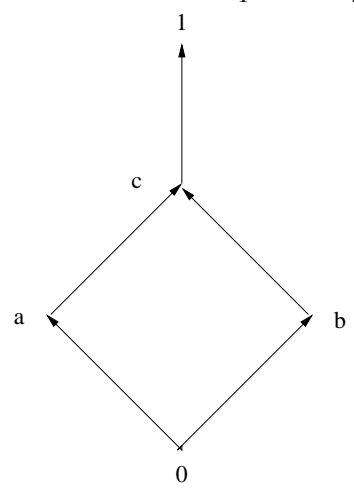

$\mathrm{HA}_{1}-\mathrm{HA}_{4}$ and $\mathrm{A}_{4}^{\mathrm{alg}}$, but not $\mathrm{HA}_{5}$.

\section{Apartness frames and duality}

We now turn to frames. The canonical frame $\mathfrak{C F}(L)$ of $L$ is the relational structure $\left\langle\operatorname{Prim}(L), \subseteq, R_{\rho}\right\rangle$, where $\langle F, G\rangle \in R_{\rho} \Longleftrightarrow F \cap \rho[G] \neq \emptyset$. An apartness frame is a structure $\langle X, \leq, R\rangle$, where $\leq$ is a partial ordering of $X$, and $R$ a nonempty binary relation on $X$, such that

$\mathrm{HF}_{0} . x \leq y$ implies $R(x) \subseteq R(y)$.

$\mathrm{HF}_{1} . R$ is irreflexive.

$\mathrm{HF}_{2} . R$ is symmetric. 
$\mathrm{HF}_{3} . R$ is co-transitive.

Relations satisfying $\mathrm{HF}_{1}-\mathrm{HF}_{3}$ are considered in theories of incomplete information, where they are called diversity relations, see e.g. [4].

Theorem 5.1. The canonical frame of a nontrivial apartness algebra is an apartness frame.

Proof. Since $\rho$ is nontrivial, there is some $x \in L, x \neq 0$ such that $\rho(x) \neq 0$. Then, there are prime filters $F, G$ such that $x \in G$ and $\rho(x) \in F$, showing that $R_{\rho} \neq \emptyset$.

$\mathrm{HF}_{0}$ : Let $F \subseteq G$, and $\langle F, H\rangle \in R_{\rho}$. Then, $F \cap \rho[H] \neq \emptyset$, and thus, $G \cap \rho[H] \neq \emptyset$.

$\mathrm{HF}_{1}$ : Since $\rho(x) \cdot x=0$ by $\mathrm{HA}_{3}, F \cap \rho[F]=\emptyset$.

$\mathrm{HF}_{2}$ : Let $F \cap \rho[G] \neq \emptyset$, say, $x \in G$ and $\rho(x) \in F$. Then, $\rho(\rho(x)) \in \rho[F]$, and $x \leq \rho(\rho(x))$ by $\mathrm{HA}_{4}$ shows that $\rho(\rho(x)) \in G$.

$\mathrm{HF}_{3}$ : Let $\langle F, G\rangle \in R_{\rho}$; we need to show that $\langle H, G\rangle \notin R_{\rho}$ implies $\langle H, F\rangle \in R_{\rho}$ for all $H \in \operatorname{Prim}(L)$. Now, $\langle F, G\rangle \in R_{\rho}$ implies that $F \cap \rho[G] \neq \emptyset$, and so there is some $x \in G$ with $\rho(x) \in F$. Suppose that $H \cap \rho[G]=\emptyset$; in particular, $\rho(x) \notin H$. Since $\rho(x)+\rho(x)^{*}=1$ by $\mathrm{HA}_{5}$, and $H$ is a prime filter, it follows that $\rho(x)^{*} \in H$. Now, $\rho(x) \in F$ implies that $\rho(\rho(x)) \in \rho[F]$, and, by $\mathrm{A}_{4}^{\text {alg }}$ and Theorem 4.3(3), $\rho(\rho(x))=\rho(x)^{*}$. This shows that $H \cap \rho[F] \neq \emptyset$, i.e. that $\langle H, F\rangle \in R_{\rho}$. Hence, $R_{\rho}$ is co-transitive.

Suppose that $\mathscr{C}(X)$ is the collection of all $S \subseteq X$ for which $S=\uparrow S$. The complex algebra $\mathfrak{C m}(X)$ of $X$ is the structure $\langle\mathscr{C}(X), \cap, \cup, \rightarrow, \emptyset, X, \rho\rangle$, where $S \rightarrow T \stackrel{\mathrm{df}}{=} \bar{\downarrow}(S \backslash T)$ and $\rho(S) \stackrel{\mathrm{df}}{=}[[R]](S)$.

Theorem 5.2. The complex algebra of an apartness frame is an apartness algebra.

Proof. By Theorem 2.1, $\langle\mathscr{C}(X), \cap, \cup, \rightarrow, \emptyset, X$,$\rangle is a complete Heyting algebra. In the next step we show that \rho$ is well defined: Let $S \in \mathscr{C}(X), x \in \rho(S)$, and $x \leq y$. By the definition of $\rho, S \subseteq R(x)$, and $\mathrm{HF}_{0}$ shows that $S \subseteq R(y)$. Thus, $y \in \rho(S)$, and it follows that $\rho(S)=\uparrow \rho(S)$.

$\mathrm{HA}_{1}$ and $\mathrm{HA}_{2}$ follows from the fact that $\rho$ is a completely co-additive sufficiency operator.

$\mathrm{HA}_{3}$ : Let $x \in \rho(S)$; then, $S \subseteq R(x)$, and the irreflexivity of $R$ shows that $x \notin S$. Hence, $\rho(S) \cap S=\emptyset$.

$\mathrm{HA}_{4}$ : Let $x \in S$; we need to show that $x \in \rho \rho(S)$, i.e. that $\rho(S) \subseteq R(x)$. Suppose that $y \in \rho(S)$; then, $S \subseteq R(y)$. $x \in S$ implies $y R x$, and the symmetry of $R$ implies that $y \in R(x)$.

$\mathrm{HA}_{5}$ : Let $x \notin \rho(S)$; then, there is some $y \in S$ such that $x \bar{R} y$. Assume that $x \notin(\rho(S))^{*}$; then, $\uparrow x \cap \rho(S) \neq \emptyset$, and thus, there is some $z \in X$ such that $x \leq z$ and $S \subseteq R(z)$. Since $x \leq z, \mathrm{HF}_{0}$ implies that $R(x) \subseteq R(z)$. Now, since $x \bar{R} y$, Lemma 2.6(2) implies that $R(x)=R(y)$, hence, $R(y) \subseteq R(z)$. It follows now from $y \in S$ and $S \subseteq R(z)$ that $z R y$, and by the symmetry of $R$ we obtain $y R z$. Hence, $z \in R(z)$, contradicting the irreflexivity of $R$.

Theorem 5.3. Suppose that $\langle X, \leq, R\rangle$ is an apartness frame, and $\langle L,+, \cdot \rightarrow, 0,1, \rho\rangle$ an apartness algebra.

1. The mapping $h: L \rightarrow \mathfrak{C m} \mathfrak{C} \mathfrak{f}(L)$ defined by $a \stackrel{h}{\mapsto}\{F \in \operatorname{Prim}(L): a \in F\}$ is an embedding of apartness algebras.

2. The mapping $k: X \rightarrow \mathfrak{C f} \mathfrak{C m}(L)$ defined by $x \stackrel{k}{\mapsto}\{S \in \mathscr{C}(X): x \in S\}$ is an embedding of apartness frames. 
Proof. 1. Clearly, $h(a)=\uparrow h(a)$, and thus, $h$ is well defined; furthermore, it is easy to see (and well known) that $h$ is injective, preserves + and $\cdot$, and $h(0)=\emptyset$ as well as $h(1)=\operatorname{Prim}(L)$.

Our next aim is to show that $h(x \rightarrow y)=h(x) \rightarrow h(y)$; recall that $h(x) \rightarrow h(y)=\overline{\downarrow(h(x) \backslash h(y))}$.

"ᄃ": Suppose that $x \rightarrow y \in F$, and assume that $F \in \downarrow(h(x) \backslash h(y))$. then, there is some $G \in \operatorname{Prim}(L)$ such that $F \subseteq G, x \in G$, and $y \notin G$. Now, $x \rightarrow y \in F \subseteq G$ and $x \in G$ imply that $x \cdot(x \rightarrow y) \in G$, and thus, $y \in G$, a contradiction.

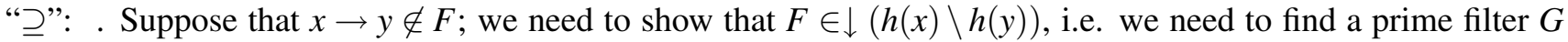
containing $F \cup\{x\}$ for which $y \notin G$; since $y \leq x \rightarrow y$, it follows that $y \notin F$. Let $F^{\prime}$ be the filter generated by $F \cup\{x\}$. Assume that $x \rightarrow y \in F^{\prime}$; then, there is some $z \in F$ such that $x \cdot z=x \rightarrow y$, and thus $x \cdot z=x \cdot(x \cdot z)=x \cdot(x \rightarrow y) \leq y$. It follows that $z \leq x \rightarrow y$. Since $z \in F$, this contradicts $x \rightarrow y \notin F$. By the Prime Ideal Theorem, there is a prime filter $G$ containing $F^{\prime}$ and disjoint from $\downarrow(x \rightarrow y)$. From $y \leq x \rightarrow y$ we obtain $y \notin G$, and therefore, $F \subseteq G \in h(x) \backslash h(y)$.

Finally, we show that $h(\rho(x))=\rho(h(x))$ :

" $\subseteq$ ": Let $F \in \operatorname{Prim}(L)$ and $\rho(x) \in F$; we need to prove that $h(x) \subseteq R_{\rho}(F)$. Thus, suppose that $x \in G$; then, $\rho(x) \in F \cap \rho[G]$, and it follows that $\langle F, G\rangle \in R_{\rho}$.

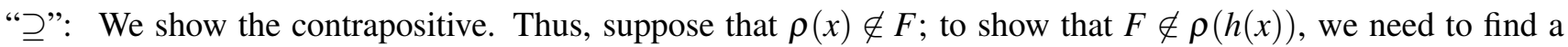
prime filter $G$ such that $x \in G$ and $F \cap \rho[G]=\emptyset$.

Let $M \stackrel{\text { df }}{=}\left\{y \in C(L): \rho\left(y^{*}\right) \in F\right\}$. Suppose that $y_{0}, \ldots, y_{n} \in M$; then, $\rho\left(y_{0}^{*}\right) \cdot \ldots \cdot \rho\left(y_{n}^{*}\right) \in F$, and

$$
\rho\left(y_{0}^{*}\right) \cdot \ldots \cdot \rho\left(y_{n}^{*}\right) \stackrel{\mathrm{HA}_{2}}{=} \rho\left(y_{0}^{*}+\ldots+y_{n}^{*}\right)=\rho\left(\left(y_{0} \cdot \ldots \cdot y_{n}\right)^{*}\right),
$$

and thus, $M$ is closed under finite products. If $y_{0} \cdot \ldots \cdot y_{n}=0$, then $y_{0}^{*}+\ldots+y_{n}^{*}=1$, since $y_{i} \in C(L)$, and thus, $y_{i}^{*} \in F$ for some $i \leq n$. This contradicts $\mathrm{HA}_{3}$, since $\rho\left(y_{i}^{*}\right) \in F$ by definition of $M$.

Next, we show that $M \cup\{x\}$ has the finite intersection property; by the preceding argument, it is sufficient to show that $y \cdot x \neq 0$ for all $y \in M$. If $y \in M$ and $y \cdot x=0$, then $x \leq y^{*}$, and therefore, $\rho\left(y^{*}\right) \leq \rho(x)$ since $\rho$ is antitone. Now, $y \in M$ implies that $\rho\left(y^{*}\right) \in F$ and it follows that $\rho(x) \in F$. This contradicts our hypothesis $\rho(x) \notin F$. Thus, $M \cup\{x\}$ has the finite intersection property and therefore the filter $M^{\prime}$ generated by $M \cup\{x\}$ is proper. Hence, by the Prime Ideal Theorem, there is a prime filter $G$ of $L$ containing $M^{\prime}$. Assume that $F \cap \rho[G] \neq \emptyset$; then, there is some $z \in G$ such that $\rho(z) \in F$, and by Lemma 4.2(3) we may suppose that $z \in C(L)$. Now, $z=z^{* *}$, and it follows that $z^{*} \in M$. Since $M \subseteq G$ and $z \in G$, we arrive at a contradiction.

2. Clearly, $k(x)$ is a prime filter of $\mathfrak{C m}(X)$. We first show that $k$ is injective: Let $x, y \in X, x \neq y$; since $\leq$ is antisymmetric, we suppose w.l.o.g. that $x \not \leq y$. Then, $y \notin \uparrow x$, and it follows that $\uparrow x \in(k(x) \backslash k(y))$. If $x \leq y$, then each $\uparrow$ closed set containing $x$ also contains $y$; thus, $k$ preserves $\leq$. Finally, let $x R y$, and set $S \stackrel{\mathrm{df}}{=} \uparrow y$; then, $S \in k(y)$, and $S \subseteq R(x)$ by $\mathrm{HF}_{0}$. Hence, $x \in[[R]](S)$, and therefore, $[[R]](S) \in k(x)$, since $[[R]](S) \in \mathscr{C}(X)$. It follows that $k(x) \cap[[R]][k(y)] \neq \emptyset$, and thus, $k(x) R_{\rho} k(y)$.

So, we have shown the duality theorem for apartness algebras:

Corollary 5.4. 1. Each apartness algebra can be embedded into the complex algebra of its canonical frame.

2. Each apartness frame can be embedded into the canonical frame of its complex algebra. 


\section{Outlook}

A discrete duality between a class Alg of algebras and a class Frm of frames leads to what is called duality via truth $[10,12]$. Algebras and frames may serve as semantic structures of formal languages and then they determine the notions of truth of formulas of a language or truth of sequents of formulas. A principle for establishing duality via truth says that a class of algebras and a class of frames provide equivalent semantics of a formal language whose signature coincides with the signature of the algebras in question. Consequently, the algebras and the frames express equivalent notions of truth.

Suppose that Alg is a class of algebras which are signature and/or axiomatic extensions of the class of lattices and define a propositional language $\mathscr{L}_{\text {Alg }}$ whose formulas are built from propositional variables of a set Var with propositional connectives that correspond to the operations of the algebras from Alg. Truth determined by Alg-semantics is defined as usual, namely, if the algebras from Alg are based on lattices with a top element 1, then for $W \in$ Alg we say that a formula $\alpha$ is true in $W$ whenever $v(x)=1$ for every evaluation $v: \operatorname{Var} \rightarrow W$, extended homomorphically to all the formulas of $\mathscr{L}_{\text {Alg. }}$. If the algebras from Alg do not have a designated top element, then usually the notion of truth applies to sequents $\alpha \vdash \beta$, where $\alpha, \beta \in \mathscr{L}_{\mathrm{Alg}}$. A sequent $\alpha \vdash \beta$ is true in an algebra $W$ if $v(\alpha) \leq v(\beta)$ for every evaluation.

Similarly, truth determined by Frm-semantics is stated in terms of models $\mathscr{M}=\langle X, m\rangle$, where $X \in \mathrm{Frm}$, and $m: \operatorname{Var} \rightarrow 2^{X}$ is a meaning function which extends homomorphically to all the formulas. A formula $\alpha$ is true in $\mathscr{M}$ whenever $m(\alpha)=X$. A sequent $\alpha \vdash \beta$ is true in $\mathscr{M}$ whenever $m(\alpha) \subseteq m(\beta)$.

If it is possible to prove that for every formula $\alpha \in \mathscr{L}_{\mathrm{Alg}}, \alpha$ is true in all models $\langle X, m\rangle$ for all $X \in$ Frm if and only if $\alpha$ is true in in the complex algebra $\mathfrak{C m}(X)$ of $X$ for every frame $X \in$ Frm, then the Representation Theorems of discrete duality enable us to establish a duality via truth between the classes Alg and Frm:

For every formula $\alpha \in \mathscr{L}_{\text {Alg }}$ the following conditions are equivalent:

1. $\alpha$ is true in all algebras $W \in$ Alg.

2. $\alpha$ is true in all models $\langle X, m\rangle$ for each $X \in$ Frm.

Along these lines we define an apartness logic whose algebraic semantics is determined by apartness algebras of Section 4, and whose frame semantics are determined by the apartness frames of Section 5. A dual tableau style deduction system for the logic will be the subject of a separate paper.

\section{References}

[1] Balbes, R. and Dwinger, P. (1974). Distributive Lattices. University of Missouri Press, Columbia.

[2] Bishop (1967). Foundations of constructive Mathematics. McGraw-Hill, New York.

[3] Bridges, D. and Vîta, L. (2003). Apartness spaces as a framework for constructive topology. Annals of Pure and Applied Logic, 119:61-83.

[4] Demri, S. and Orłowska, E. (2002). Incomplete Information: Structure, Inference, Complexity. EATCS Monographs in Theoretical Computer Science. Springer-Verlag, Heidelberg.

[5] Düntsch, I. and Orłowska, E. (2000). Beyond modalities: Sufficiency and mixed algebras. In Orłowska, E. and Szałas, A., editors, Relational Methods in Computer Science Applications, pages 277-299, Heidelberg. Physica Verlag. 
[6] Düntsch, I. and Vakarelov, D. (2007). Region-based theory of discrete spaces: A proximity approach. Annals of Mathematics and Artificial Intelligence, 49:5-14.

[7] Ishihara, H., Mines, R., Schuster, P., and Vîta, L. (2006). Quasi-apartness and neighbourhood spaces. Annals of Pure and Applied Logic, 141:296-306.

[8] Jónsson, B. and Tarski, A. (1951). Boolean algebras with operators I. 73:891-939.

[9] Naimpally, S. A. and Warrack, B. D. (1970). Proximity Spaces. Cambridge University Press, Cambridge.

[10] Orłowska, E. and Rewitzky, I. (2005). Duality via Truth: Semantic frameworks for lattice-based logics. Logic Journal of the IGPL, 13(4):467-490.

[11] Orłowska, E. and Rewitzky, I. (2007). Discrete duality and its applications to reasoning with incomplete information. volume 4585 of Lecture Notes in Artificial Intelligence, pages 51-56. Springer.

[12] Orłowska, E., Rewitzky, I., and Düntsch, I. (2005). Relational semantics through duality. In MacCaull, W., Winter, M., and Düntsch, I., editors, Proceedings of the $8^{t} h$ International Conference on Relational Methods in Computer Science and the $3^{r}$ International Workshop on Applications of Kleene Algebra and Workshop of COST Action 274, volume 3929 of Lecture Notes in Computer Science, pages 17-32, Heidelberg. SpringerVerlag.

[13] Priestley, H. A. (1972). Ordered topological spaces and the representation of distributive lattices. Proc. London Math. Soc., 3:507-530.

[14] Richman, F. (2002). Removing inequality. Unpublished.

[15] Vakarelov, D., Dimov, G., Düntsch, I., and Bennett, B. (2002). A proximity approach to some region-based theories of space. J. Appl. Non-Classical Logics, 12:527-529.

[16] Vîta, L. (2005). On complements of sets and the Efremovic condition in pre-apartness spaces. J. UCS, 11(12):2159-2164. 